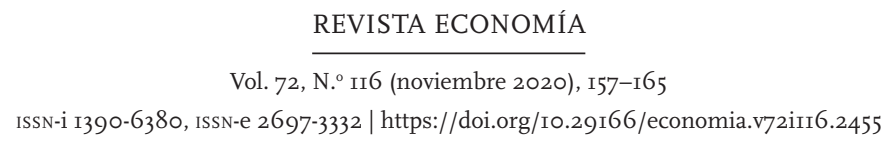

\title{
CUENTA SATÉLITE DE TECNOLOGÍAS DE INFORMACIÓN Y COMUNICACIÓN DEL ECUADOR, AÑO 2015
}

\author{
THE SATELLITE ACCOUNT OF INFORMATION AND \\ COMMUNICATION TECHNOLOGY FOR ECUADOR 2015 \\ ROBERTHO ROSERO E., ${ }^{\mathrm{I}}$ CARLOS ROMERO, ${ }^{\mathrm{I}}$ GONZALO SILVA ${ }^{2}$ \\ ${ }^{1}$ Universidad Central del Ecuador (Ecuador) \\ ${ }^{2}$ Investigador independiente
}

Recepción del manuscrito: 19 de septiembre de 2020

Aceptación versión final: 21 de noviembre de 2020

\begin{abstract}
Resumen La Cuenta Satélite de Tecnologías de la Información y Comunicación para el Ecuador, año 2015, establece un estudio pormenorizado de las actividades económicas características, conexas y transacciones económicas de este importante sector de la economía ecuatoriana. En la investigación planteada, se describe la metodología utilizada, en la cual se delimita los productos y las industrias del sector de las Tecnologías de la Información y Comunicación (TIC). Al final, se muestran los resultados obtenidos producto de las tablas oferta-utilización, los distintos agregados macroeconómicos, como el valor agregado y el PIB, los cuales permiten evaluar la contribución e importancia económica del sector TIC a la economía nacional durante el 2015.
\end{abstract}

PALABRAS CLAVE Cuentas nacionales, cuenta satélite, tecnologías de la información y comunicación.

ABSTRACT The Satellite Account of Information and Communication Technology for Ecuador 2015, establishes a detailed study of the characteristic economic activities, related and economic transactions of this important sector of the Ecuadorian economy. This research describes the methodology used, which defines the products and industries of the Information and Communication Technology (ICT) sector. In the end, the results obtained from the supplyuse tables, the different macroeconomic aggregates, such as value added and GDP, are shown, which allow to assess the contribution and economic importance of the ICT sector to the national economy during 2015.

KEYWORDS National accounts, satellite account, information and communication technologies. JEL CODES E01, L8, L86. 


\section{ANTECEDENTES}

La elaboración de la Cuenta Satélite de Ecuador del sector de las Tecnologías de Información y Comunicación (CSE-TIC), año 2015, se enmarca como un aporte al conocimiento económico del sector de las telecomunicaciones del país, con el propósito de contar con las herramientas adecuadas para la toma de decisiones de los actores económicos de este importante sector. La implementación de cuentas satélites para el sector de las (TIC) es un proceso que se viene gestionándose en los países de América Latina desde hace varios años. En este sentido, desde octubre de 2018, la Universidad Central del Ecuador, a través de un proyecto de investigación, se propone la elaboración de la cuenta satélite con el ánimo de generar agregados macroeconómicos que ayuden a la comprensión de carácter económico de este importante sector.

\section{MARCO CONCEPTUAL}

El marco conceptual de la CSE-TIC tiene como referencia bibliográfica básica la metodología de elaboración de las cuentas nacionales publicada en el Sistema de Cuentas Nacionales 2008 ( $\mathrm{SCN}$ ) elaborado por Naciones Unidas y otras organizaciones; y como referencia bibliográfica específica la publicación Classifying information and communication technology services realizada por la Organización para Cooperación y Desarrollo Económicos (OCDE) en 2006.

\section{CUENTAS NACIONALES}

El SCN es el conjunto de recomendaciones internacionales relativas a una combinación de conceptos, definiciones, clasificaciones y reglas de registro contables acordadas para medir la actividad económica de acuerdo con convenciones contables estrictas, basadas en principios económicos. El scN sirve de apoyo al análisis económico, la toma de decisiones y la formulación de políticas públicas. El objetivo esencial del sCN es ofrecer un marco conceptual y contable para crear estadísticas macroeconómicas apropiadas para el análisis y la evaluación de la estructura productiva, la formulación de políticas públicas y la toma de decisiones.

El uso más común del scN es el seguimiento del comportamiento de la economía. Los resultados obtenidos de las cuentas nacionales, desagregados por actividades económicas y unidades institucionales, facilitan el análisis de las principales transacciones económicas de bienes y servicios, distributivas y financieras, así como de los stocks de activos financieros y no financieros, los pasivos y otros flujos económicos. Además, el sCN ofrece información sobre los agregados económicos y relaciones clave que solo pueden obtenerse y medirse dentro de un marco contable.

Finalmente, el SCN sirve de marco para la coordinación de las estadísticas económicas según dos ópticas. Desde el punto de vista conceptual, se garantiza la coherencia de los conceptos, definiciones y clasificaciones en los diferentes sistemas estadísticos y, desde el punto de vista contable, garantiza la coherencia numérica de datos procedentes de distintas fuentes estadísticas.

\section{CUENTAS SATÉLITES}

Las cuentas satélites son una extensión del sistema de cuentas nacionales y constituyen una herramienta de medición económica que describen en detalle la estructura y comportamiento de 
Tabla 6. Producción en la CSE-TIC 2015

\begin{tabular}{|c|c|c|c|}
\hline CPTIC & Descripción & PCC TOTAL & $\begin{array}{c}\text { Valor } \\
\text { porcentual }\end{array}$ \\
\hline TIC01001 & Maquinarias de uso no doméstico TICS & 0 & $0,00 \%$ \\
\hline TIC01002 & Dispositivos electrónicos para uso contable y financiero & 374 & $0,01 \%$ \\
\hline TIC01003 & Ordenadores y accesorios electrónicos & 546 & $0,01 \%$ \\
\hline TIC01004 & Dispositivos eléctricos & 145.702 & $2,77 \%$ \\
\hline TIC01005 & Dispositivos de transmisión de radio, tv y comunicaciones & 17.104 & $0,33 \%$ \\
\hline TIC01006 & Equipos diversos en TICS & 350 & $0,01 \%$ \\
\hline TIC02001 & Comercio & 536.220 & $10,19 \%$ \\
\hline TIC03001 & Servicios de administración y rectoría pública en TICS & 43.450 & $0,83 \%$ \\
\hline TIC03002 & Otros servicios de administración pública en TICS & 30.050 & $0,57 \%$ \\
\hline TIC04001 & Servicios de telefonía fija & 539.164 & $10,25 \%$ \\
\hline TIC05001 & Servicios de telefonía celular & 2.628 .218 & $49,96 \%$ \\
\hline TIC06001 & Servicios de internet & 534.742 & $10,17 \%$ \\
\hline TIC07001 & $\begin{array}{l}\text { Servicios de distribución, publicación } \\
\text { y edición de programas, audio y video }\end{array}$ & 39.115 & $0,74 \%$ \\
\hline TIC08001 & Servicios de investigación y desarrollo & 64 & $0,00 \%$ \\
\hline TIC09001 & Reparación, mantenimiento y limpieza & 136.391 & $2,59 \%$ \\
\hline TIC09002 & Leasing de TICS & 2.192 & $0,04 \%$ \\
\hline TIC09003 & Servicio TICS especializados en contabilidad & 35.942 & $0,68 \%$ \\
\hline TIC09004 & Servicios de arquitectura e ingeniería para TICS & 26.136 & $0,50 \%$ \\
\hline TIC09005 & Otros servicios & 274.611 & $5,22 \%$ \\
\hline TIC10001 & Servicios conexos & 269.963 & $5,13 \%$ \\
\hline TOTAL & & 5.260 .334 & $100 \%$ \\
\hline
\end{tabular}

Elaboración y fuente: propia.

ciertas áreas socioeconómicas especificas en base a los conceptos, definiciones, clasificaciones y reglas de registro contable del marco central de las cuentas nacionales de una manera detallada y flexible, sin sobrecargar o distorsionar el sistema central.

Elaborar cuentas satélites presenta ciertas ventajas. En primer lugar, ofrecen un alto grado de robustez en el proceso de elaboración, ya que las definiciones y conceptos centrales utilizados son los del SCN. En segundo lugar, las cuentas corrientes de acumulación y balances de los sectores institucionales y las tablas de oferta-utilización de bienes y servicios garantizan la consistencia y la coherencia de sus resultados. Finalmente, los resultados de la cuenta satélite pueden analizarse en relación con los agregados de la economía, permitiendo estimar la contribución del ámbito de estudiado al crecimiento de la economía.

\section{METODOLOGÍA DE LA CUENTA SATÉLITE DE TECNOLOGÍAS}

DE INFORMACIÓN Y COMUNICACIÓN (CS-TIC)

La CS-TIC del Ecuador 2015 se elabora en base a los lineamientos metodológicos del marco central del SCN 2008. Se describe de manera amplia y detallada la actividad del sector TIC, de esta 
manera se cuantifican todos los aspectos característicos que las cuentas nacionales las describe de forma resumida. La CS-TIC es un instrumento técnico cuantitativo para valorar la producción de bienes y servicios de TIC y su aporte al producto interno bruto (PIB). Esto facilita el diseño y formulación de políticas públicas en beneficio del desarrollo del sector en cuestión.

La cuantificación satélite de cualquier sector, actividad o función económica exige una delimitación. Es necesario identificar los bienes y servicios característicos del sector TIC. Esto permite establecer las actividades que los producen. La definición de los bienes y servicios característicos de tecnologías de información y comunicación se basa en las recomendaciones de la OCDE con el fin de facilitar la comparación de los agregados TIC.

Según la OCDE, un bien es característico TIC cuando cumple la función de procesar y comunicar información utilizando procesos electrónicos para detectar, medir o recoger fenómenos físicos o controlar procesos físicos. Por otro lado, un servicio característico debe facilitar el procesamiento y comunicación de información por medios electrónicos. La Cs-TIC tiene como objetivo analizar la información estadística de base y cuantificar, mediante la aplicación de la metodología de elaboración del scN, la actividad productiva, el comportamiento y desenvolvimiento del sector de las TIC en la actividad económica del Ecuador. Las estadísticas disponibles relacionadas con las CS-TIC permiten, con la aplicación de la metodología del sCN, llevar a cabo el proceso de elaboración en las siguientes etapas. Primero, delimitar el campo de acción de las actividades de TIC. Luego, especificar las nomenclaturas de actividades o industrias de TIC y de productos característicos de TIC. Después, se elabora los equilibrios de oferta y utilización de bienes y servicios característicos Tic, a partir de la cuenta de producción de las industrias Tic. Finalmente, se calcula el рів del sector тіс.

\section{CLASIFICADORES DE LA CUENTA SATÉLITE DE TECNOLOGÍAS DE INFORMACIÓN Y COMUNICACIONES}

Establecer los productos e industrias de la CS-TIC es de suma importancia, pues en esta definición se basa todo el análisis posterior. La forma de determinar los productos se basa en el Clasificador Central de Productos (CPC) de las Naciones Unidas y el Clasificador de Productos de la Cuentas Nacionales (CPCN) del Banco Central del Ecuador (BCE) y sus correspondencias con el Sistema Armonizado utilizado en la clasificación de bienes en el comercio exterior. Para determinar las actividades pertinentes se utiliza el Clasificador Internacional Industrial Unificado (CIIU) de las Naciones Unidas y el Clasificador de Industrias de las Cuentas Nacionales (CICN) del вCE (ver tablas 1 y 2 del anexo). ${ }^{1}$ En este sentido, para definir los productos e industrias de la CS-TIC, se toma como base la definición presentada del sector TIC, párrafos anteriores. El CIIU contiene veintiuna secciones, para la elaboración de la Cs-TIC se han seleccionado ocho. Estas ocho secciones tienen como referencia la información obtenida del Ministerio de Telecomunicaciones y de la Sociedad de la Información (MINTEL) además de OCDE (ver tabla 3 del anexo). El clasificador de productos de la Cs-TIC está constituido de 1259 partidas del CPC y 22 del CPCN.

El código del clasificador está conformado por dos niveles, el primer nivel hace referencia a la industria o actividad con el que se encuentra relacionado el producto y el segundo nivel corresponde al producto, bien o servicio (ver ilustración 1 del anexo). El clasificador de productos contiene veinte registros (ver tabla 4 del anexo) estructurados en tres grupos: el primer 
Tabla 7. Impuestos sobre productos. CSE-TIC y economía total, 2015

\begin{tabular}{lrrr}
\hline Impuestos sobre productos & $\begin{array}{l}\text { Valor total de } \\
\text { la economía }\end{array}$ & Valor CSTIC & $\begin{array}{r}\text { Relación porcentual CSTIC } \\
\text { sobre la economía total }\end{array}$ \\
\hline Impuesto al valor agregado & 6.352 .338 & 764.971 & $12,04 \%$ \\
\hline Derechos arancelarios & 1.950 .791 & 339.883 & $17,42 \%$ \\
\hline Subsidios sobre productos & 1.916 .536 & 0 & $0,00 \%$ \\
\hline Impuestos indirectos sobre productos & 861.283 & 56.780 & $6,59 \%$ \\
\hline
\end{tabular}

Elaboración y fuente: propia.

grupo corresponde a bienes TIC, el segundo grupo a las actividades comerciales del sector y el tercer grupo a servicios TIC. El clasificador de actividades contiene diez registros.

También se utilizaron clasificadores de comercio exterior como la Clasificación Uniforme para el Comercio Internacional (CUCI), el Sistema Armonizado (HS, por sus siglas en inglés) y la Nomenclatura Arancelaria de la Comunidad Andina de Naciones (NANDINA) que es una desagregación específica del Hs para Comunidad Andina de Naciones.

Cada registro del clasificador de productos de la Cs-TIC se encuentra relacionado de manera excluyente con un registro del CPCN, y éstos a su vez están relacionados con registros específicos del CPC. En el clasificador de industrias se ha desagregado el registro del CICN, comunicaciones e información, en las industrias de telefonía fija, móvil y de internet, dada la relevancia de estos sectores (ver tabla 2 del anexo).

Los clasificadores de productos y de actividades de la CS-TIC definen la estructura básica de la tabla de oferta y la tabla de utilización. Es por esta razón que los productos se encuentran relacionados de manera unívoca a una industria. Como ya se explicó anteriormente, el primer nivel de la codificación de los clasificadores de productos de la Cs-Tic define a qué industria está relacionado cada producto (ver tabla 5 del anexo). El clasificador permite continuar con el cálculo de los campos de la tabla oferta utilización y otros agregados macroeconómicos del sector Tic.

\section{RESULTADOS}

En esta sección, se describen los resultados obtenidos en la CS-TIC. Se presentan, en primer lugar, los resultados de la oferta y, posteriormente, los resultados de la utilización. Finalmente, se presenta el PIB del sector TIC. En otras palabras, la representación del sector TIC en la economía ecuatoriana en el 2015.

\section{OFERTA CS-TIC}

Las importaciones ascienden a USD 1388 millones dentro del sector TIC para el año 2015. Este valor está conformado principalmente por las importaciones de dispositivos para comunicaciones que representa el $62 \%$ del total, seguido por la importación de ordenadores con el $24 \%$ del total y dispositivos eléctricos con el $11 \%$ del total. Combinados, esto tres productos representan el 95\% del monto de importaciones. Los rubros de equipos diversos, software, dispositivos 
para uso contable y maquinarias de uso no doméstico representan valores menores o iguales que el 1\% (ver ilustración 2 en el anexo). El valor de las importaciones del sector Tic representa el 6,73\% de las importaciones totales de la economía para el año 2015.

En el sector TIC, la producción de servicios es superior a la producción de bienes. La producción de servicios es de USD 5096 millones mientras que la producción de bienes es de USD 164 millones (ver ilustración 3 del anexo).

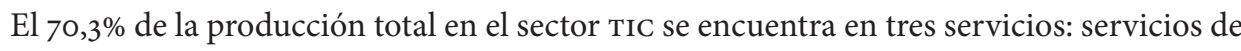
telefonía celular, los de telefonía fija, y los de internet. De estos servicios, el de telefonía celular es el que más aporta al total de la producción con el 50\%. Los servicios de investigación y desarrollo TIC, es el servicio con menor valor de producción, el cual representa el o,001\% de la producción total de la cuenta satélite. El valor de producción de bienes TIC, como ordenadores, dispositivos electrónicos, entre otros es menor en relación con los servicios prestados. Estos productos representan el o,03\% del valor de la producción total.

El mayor valor de producción de bienes dentro del sector TIC se encuentra en el rubro de dispositivos eléctricos, como cables de energía que están dentro de dispositivos electrónicos o cables de transmisión de datos, el valor de estos bienes representa el 2,77\% del total (ver Tabla 6).

El valor agregado bruto de la industria en el 2015 fue aproximadamente USD 2545 millones. La industria servicios de telefonía celular es la que más aporta al valor agregado bruto con alrededor de USD 1410 millones. Las industrias de servicios de telefonía fija y de servicios de internet se ubican en segundo lugar de contribución con USD 315 y USD 288 millones, respectivamente. La industria de fabricación de bienes del sector es la que menos aporta al valor agregado con USD 75 millones, juntamente con la industria de comercio de bienes TIC, las actividades de publicación de programas y las actividades de investigación y desarrollo (ver ilustración 4 del anexo).

Los derechos arancelarios en el sector TIC suman USD 339 millones. Los impuestos indirectos suman USD 56 millones. La recaudación por el impuesto al valor agregado es de USD 764 millones. No existen subsidios en el sector TIC. En total, el monto de impuestos sobre productos suma en total USD 1161 millones. Al comparar los valores de los impuestos de la CS-TIC con el total de la economía, se puede observar que el valor del IVA del sector TIC representa el 12\% del total de la economía, los derechos arancelarios $17 \%$ del total de la economía y $6 \%$ de los impuestos indirectos (ver Tabla 7 ).

La oferta total a precios de comprador es de USD 7810 millones. La oferta se compone en un $38 \%$ de los servicios de telefonía celular, $33 \%$ de bienes del sector TiC, un $9 \%$ por otros servicios - leasing, servicios especializados para ingeniería en TIC, entre otros-, un $8 \%$ por servicios de internet, de igual manera en $8 \%$ para servicios de telefonía fija, $2 \%$ en servicios de reparación y mantenimiento y $1 \%$ por servicios de software. Los servicios de telefonía celular incluyen los servicios de voz móvil y los servicios de datos móviles, es decir, el acceso a internet por un dispositivo celular esta medido dentro del rubro servicios de telefonía celular (ver ilustración 5 del anexo). 
Tabla 8. Resumen oferta-utilización CSTIC, miles de dólares

\begin{tabular}{lrlr}
\hline Oferta & & Utilización & \\
\hline Importaciones & 1.388 .134 & Consumo intermedio & 2.169 .406 \\
\hline Producción & 5.260 .334 & GCFHg & 4.574 .767 \\
\hline Impuestos indirectos & 56.780 & Gasto del gobierno & 73.500 \\
\hline IVA & 764.971 & FBK & 947.763 \\
\hline Derechos arancelarios & 339.883 & Variación de Existencias & -6.242 \\
\hline Subsidios & 0 & Exportaciones & 50.908 \\
\hline Oferta total & 7.810 .102 & Utilización total & 7.810 .102 \\
\hline
\end{tabular}

Elaboración y fuente: propia.

El total de la oferta a precios de comprador, USD 7810 millones, se distribuye en la utilización de la siguiente manera: el gasto de consumo final de los hogares (GCFHg) representa el 58\% de la utilización, el consumo intermedio representa el 28\%, la formación bruta de capital representa el $12 \%$ y las exportaciones y el gasto de gobierno representan el $1 \%$ cada uno (ver ilustración 6 del anexo).

El total del consumo intermedio es de USD 2169 millones, donde el $24 \%$ corresponde a telefonía celular. En segundo lugar, se encuentra el rubro de equipos para comunicaciones con el $17 \%$, seguido de servicios conexos con el $14 \%$, ordenadores con el $11 \%$, telefonía fija con el $9 \%$ y servicios de internet con el 6\%. El resto de productos representa el 19\% restante, en donde se encuentran servicios especializados y otros como servicios de limpieza y software (ver ilustración 7 del anexo).

El total de GCFHg es de USD 4574 millones. El 54\% se dirige principalmente hacia los servicios de la telefonía celular. El 13\% se dirige hacia dispositivos de comunicación. El 10\% se dirige hacia los servicios de internet, el $9 \%$ a servicios de telefonía fija, el $6 \%$ en dispositivos eléctricos y el $8 \%$ en el resto de los servicios (ver ilustración 8 del anexo).

El gasto de gobierno en el sector TIC se compone por el servicio de la administración pública que realizan tres entidades que son rectoras dentro del sector. Estas instituciones son el mintel, la Agencia de Regulación de las Telecomunicaciones (ARCOTEL) y la Dirección Nacional de Registro de Datos Públicos (DINARDAP). Se trata de un gasto colectivo y que asciende a UsD 73 millones.

El monto de formación bruta de capital fijo es de UsD 947 millones. Los dispositivos utilizados para la comunicación como celulares, radios, televisores, etc., concentran el $60 \%$ de la formación bruta de capital fijo. En segundo lugar, se encuentra a ordenadores y otros accesorios electrónicos que representa el 35\%. Por último, la formación bruta de capital fijo se distribuye un $5 \%$ en dispositivos eléctricos y un $3 \%$ en otros bienes y servicios (ver ilustración 9 del anexo).

La variación de existencias indica la reducción o acumulación del stock de existencias. De acuerdo con los cálculos realizados en la CS-TIC, existe una reducción de existencias de USD 6,2 millones. Esta disminución se explica por los rubros de dispositivos de comunicaciones y dispositivos diversos que registran una disminución de USD 8,4 millones. En los demás productos de la CS-TIC se registra en total un aumento del stock de USD 2,2 millones. 
Las exportaciones de productos del sector TIC es de USD 50,9 millones. El 70\% se concentra en dispositivos eléctricos utilizados únicamente por bienes TIC. Se exportaron UsD 8,9 millones en dispositivos utilizados para comunicaciones y USD 5 millones en ordenadores, los cuales representan el 17\% y el $9 \%$ respectivamente del total de las exportaciones. Al comparar las importaciones y las exportaciones específicas del sector, se puede observar que existe un déficit USD 1337 millones (ver ilustración 10 del anexo). En la tabla resumen se pueden observar todos los agregados de la Cs-Tic, de tal manera que se puede comprobar la igualdad entre la oferta y la utilización (ver Tabla 8).

\section{PIB DEL SECTOR TIC}

Existen tres métodos para calcular el PIB. El método del ingreso, el método del gasto y el método de la producción. El PIB en la Cs-TiC se lo define a través del método de la producción. Este método consiste en la sumatoria del valor agregado de cada industria más los impuestos sobre productos y menos las subvenciones. El total del PIB de la CS-TIC por este método es de USD 3707 millones. Este valor representa el 3,73\% de la economía total. El PIB de la economía en el 2015 a precios corrientes fue de USD 99.290 millones.

\section{NOTA}

$1 \mathrm{El}$ anexo al que se hace referencia para tablas e lustraciones se lo puede hallar en https://cutt.ly/tjtmvio

\section{REFERENCIAS}

Agencia de Regulación y Control de las Telecomunicaciones (ARCOTEL). (2019). Estadísticas de telecomunicaciones. http://www.arcotel.gob.ec/estadisticas-de-telecomunicaciones2/

Banco Central del Ecuador (вСЕ). (2007). Clasificaciones en la contabilidad nacional ecuatoriana. Banco Central del Ecuador. https://www.bce.fin.ec/index.php/informacioneconomica/sector-real

Banco Central del Ecuador (вСE). (2019). Cuentas Nacionales. Tablas oferta utilización nivel 2 (2007-2015). https://www.bce.fin.ec/index.php/component/k2/item/763-cuentas-nacionales

Banco Central del Ecuador (вСЕ). (2019). Estadísticas de comercio exterior. https://www.bce.fin.ec/index. $\mathrm{php} / \mathrm{c}$-exterior

Comisión Económica para la América Latina y el Caribe (CEPAL). (1972). Estudios e investigaciones. https:// repositorio.cepal.org/bitstream/handle/11362/29196/S6500320_es.pdf?sequence=1\&isAllowed=y

Comtrade. (2019). Trade statistics. https://comtrade.un.org/data/

Germán, P. B. (2008). Tecnologías de Información y Comunicación en la República Dominicana: Una primera aproximación. Unidad Asesora de Análisis Económica y Social.

Instituto Nacional de Estadística y Censos (INEC). (2015). Encuesta de Condiciones de Vida 6r. https:// www.ecuadorencifras.gob.ec/documentos/web-inec/ECV/ECV_2015/

Instituto Nacional de Estadística y Censos (INEC). (2015). Encuesta de manufactura y minería. https:// www.ecuadorencifras.gob.ec/manufactura-y-mineria/

Ministerio de Economía de Chile, I. N. (2005). Cuenta satélite de tecnologías de información y comunicación en Chile. (s. d.). 
Naciones Unidas. (2006). Clasificación Industrial Internacional Uniforme de todas las actividades económicas (CIIU). División de Estadística.

Naciones Unidas. (2015). Clasificación central de productos. División estadística.

Naciones Unidas, C. E. (2008). Sistema de cuentas nacionales 2008. CEPAL.

Organización para la Cooperación y el Desarrollo Económicos (OCDE). (2007). Classifying information and communication technology (ICT) services.(s. d.).

Servicio de Rentas Internas (SRI). (2019). Estadísticas generales de recaudación. https://www.sri.gob.ec/ web/guest/estadisticas-generales-de-recaudacion

Superintendencia de Compañías, valores y seguros. (2019). Portal de información. https://appscvsmovil. supercias.gob.ec/portalInformacion/sector_societario.zul 